These articles have been accepted for publication in the British Journal of Dermatology and are currently being edited and typeset. Readers should note that articles published below have been fully refereed, but have not been through the copy-editing and proof correction process. Wiley-Blackwell and the British Association of Dermatologists cannot be held responsible for errors or consequences arising from the use of information contained in these articles; nor do the views and opinions expressed necessarily reflect those of Wiley-Blackwell or the British Association of Dermatologists

Accepted Date : 13-Aug-2012

Article type : Original Article

\title{
The distribution pattern of Segmental Vitiligo: clues for somatic mosaicism
}

\author{
N. van Geel ${ }^{1 *}$, R. Speeckaert ${ }^{1 *}$, E. Melsens ${ }^{1}$, S.P. Toelle ${ }^{2}$, M. Speeckaert ${ }^{3}$, S. De Schepper ${ }^{1}$, J. \\ Lambert $^{1}$, L. Brochez ${ }^{1}$ \\ ${ }^{1}$ Department of Dermatology, Ghent University Hospital, De Pintelaan 185, 9000 Ghent, Belgium \\ ${ }^{2}$ Department of Neurology, University Children's Hospital Zurich, Steinwiesstrasse 75,8032 Zurich, \\ Switzerland
}

${ }^{3}$ Department of Internal Medicine, Ghent University Hospital, De Pintelaan 185, 9000 Ghent, Belgium

*Equally contributed

\section{Correspondence:}

Nanja van Geel, MD, PhD

Department of Dermatology, Ghent University Hospital

De Pintelaan 185, 9000 Ghent

Belgium

Tel: + 32-93322298; Fax: + 32-93324996 
Nanja.vangeel@UGent.be

This research was conducted at the Department of Dermatology, Ghent University Hospital, Belgium

Running head: Distribution pattern Segmental Vitiligo

The authors have no conflict of interest.

Funding sources: This research was supported by a research grant to N. van Geel from the Scientific

Research Foundation-Flanders (FWO Senior Clinical Investigator) and to R. Speeckaert from the Research Foundation (No. BOF10/doc/403), Ghent University.

\section{Bulleted statements}

\section{What's already known about this topic?}

The etiopathogenesis of segmental vitiligo is still controversial. For many years it has been stated that it has a dermatomal distribution. More recently similarities with a Blaschkoid pattern have been reported. So far, the distribution pattern of segmental vitiligo has not been compared in a systematic way to other unilateral band shaped dermatoses with a possible mosaic or neurogenic background.

\section{What does this study add?}

This study provides additional evidence that segmental vitiligo has been classified incorrectly in the past to a dermatomal distribution. We demonstrate that some mosaic dermatoses of melanocytic origin (such as segmental lentiginosis) can have a clear clinical overlap with segmental vitiligo. This observation supports the possible role of cutaneous mosaicism for at least a part of the segmental vitiligo lesions. 


\begin{abstract}
Background: Segmental vitiligo is characterized by a unilateral and localized distribution. So far, the underlying mechanism is still an enigma.
\end{abstract}

Objective: To get insight in the etiopathogenesis of segmental vitiligo by comparing the distribution pattern of dermatoses with a possible mosaic or neurogenic background to segmental vitiligo.

Methods: In this retrospective observational study the distribution pattern of 724 unilateral, linear or band shaped control lesions was compared to 181 segmental vitiligo lesions. Clinical photographs were used to score similarities according to a defined grading system (scale 0 for no similarities up to 4 for complete similarity). Control lesions were evaluated both individually and after grouping into different cell types.

Results: In general, only in a minority of cases (36.9\%), similarities (grade 1-4) were seen between control lesions and segmental vitiligo. Grade 2-4 similarities were mainly seen in segmental lentiginosis $(73.7 \%)(\mathrm{P}<0.001)$. The best grade for correspondence (grade 3 to 4$)$ was only significantly more observed in segmental lentiginosis $(36.8 \%$ vs $3.5 \%, \mathrm{P}<0.001)$ and epidermal naevus verrucosus $(12.5 \%$ vs $3.7 \%, \mathrm{P}=0.008)$ compared to the other control lesions. The distribution pattern of segmental vitiligo was significantly overlapping those of other disorders originating from melanocytes.

Conclusion: Our results demonstrate that the distribution pattern of segmental vitiligo is not entirely similar to another skin disease, although some mosaic skin disorders have more overlap with segmental vitiligo compared to others. The remarkable clinical similarity with several cases of mosaic diseases involving melanocytes support the hypothesis that cutaneous mosaicism may be involved in segmental vitiligo.

Key words: vitiligo, segmental vitiligo, lines of Blaschko, halo naevi, cutaneous mosaicism 


\section{Introduction}

Segmental vitiligo, a subtype of vitiligo, is characterized by its early onset, rapid stabilization and unilateral distribution. Epidemiological information on this condition remains rather limited, compared to the non-segmental type of vitiligo. The etiopathogenesis of segmental vitiligo is still unclear, although new insights have been described more recently. ${ }^{1}$

In an observational study by our group different phenotypes of segmental vitiligo were found, in which segmental lesions can be present uni- and bilateral and with or without halo naevi. ${ }^{2}$ In this study it was also observed that the majority of segmental vitiligo lesions did not fit exactly within the borders of the commonly mentioned 'dermatomal' lines. We therefore hypothesized that in the past segmental vitiligo could have been confined incorrectly to these lines. However, a typical unique and recurring pattern often with a very clear midline demarcation has been observed. So far, this distribution pattern is poorly understood. One hypothesis involves neural mechanisms (e.g. sympathic nerve function, neuropeptides) as vitiligo was originally believed to have a dermatomal distribution. ${ }^{3-5}$ A second theory is that segmental vitiligo lesions represent a vulnerable subpopulation of melanocytes

as seen in cutaneous mosaicism. ${ }^{1,6-8}$ To our knowledge, so far, no observational studies compared the distribution characteristics of a high number of segmental vitiligo lesions to other unilateral distributed dermatoses in a systematic way. This study aims to analyze this segmental vitiligo pattern in more detail and to search for clinical evidence that support current hypotheses with respect to the etiopathogenesis of segmental vitiligo.

The primary purpose of the current study was to determine the similarities in distribution compared to other dermatoses with a possible link to cutaneous mosaicism or a neurogenic background. Additionally, we evaluated the possible differences in distribution patterns between segmental vitiligo with and without associated halo naevi or congenital naevi.

The clinical rationale for this study is that it can lead to more insights in the etiopathogenesis of segmental vitiligo. 


\section{Material and methods}

\section{Segmental vitiligo patients}

Patients, diagnosed at the department of Dermatology, Ghent University Hospital, between 2004-2011 as having segmental vitiligo, were invited to participate in this study. This study was approved by the local ethics committee of the Ghent University Hospital. Data were obtained in a retrospective setting. All patients were asked to sign an informed consent for the use of their medical data including also clinical data with respect to presence, location and number of halo naevi. These data were based on clinical examination of the whole body with additional Wood's light. In some segmental vitiligo patients, the 'opting out' system was applicable i.e. patients did not refuse the use of their data from the available medical files. This opting out system was also used for the control group, including patients seen at our department between 2004 and 2011 with unilateral distributed dermatoses, band shaped dermatoses or dermatoses possibly linked to cutaneous mosaicism according to Happle et al. ${ }^{9}$

\section{Control group}

Dermatoses eligible for inclusion in the control group were: herpes zoster, capillary malformations (vascular naevi), verrucous epidermal naevus, ILVEN, naevus sebaceus, phacomatosis pigmentokeratotica, macular hyperpigmentation (including linear and whorled naevoid hypermelanosis, café au lait macules, isolated macular hyperpigmentations), lentiginous lesions (including naevus spilus, segmental lentiginosis), Becker's naevus, segmental neurofibromatosis type 1, naevoid hypomelanosis (including previously called "hypomelanosis of Ito", naevus depigmentosus), incontinentia pigmenti, CHILD syndrome, cutaneous leiomyomatosis, segmental Darier's disease, segmental Hailey-Hailey disease, linear lichen planus, linear lichen sclerosus, linear scleroderma, lichen striatus and linear porokeratosis. These disorders were subsequently also classified 
according to the primary affected cell type (keratinocytes, melanocytes, fibroblasts, nerve cells, endothelial cells, smooth muscle cells) in each condition. As such, segmental vitiligo could be compared to the distribution patterns observed in each cell type in order to gain more insights into the underlying mechanisms of segmental vitiligo. To increase the number of control lesions, additional sources were used, including pictures published in medical literature (books, journals) and pictures free available online. Only lesions with a unilateral distribution or more or less segmental, band shaped or linear distribution were included.

\section{Camparison analysis}

Photographs of all control lesions were used to evaluate the pattern of lesions and to estimate the possible overlap in distribution with the segmental vitiligo lesions. All segmental vitiligo lesions were in addition traced with red ink on a blank standardized body chart (contours redrawn from Happle R, with permission ${ }^{9,10}$ ) on transparent paper directly from the digital pictures (with the help of a zoom in and out function). This was used as a help during the assessment and to evaluate the possible existence of a recurring segmental vitiligo pattern in a more standardized way.

Before analyzing the data a differentiation between the different body localizations was made. Furthermore, a sub-evaluation was made between segmental vitiligo lesions without and with associated halo naevi or congenital naevi.

Based on the clinical presentation, all control lesions were evaluation on 4 different aspects/items: 1) the shape of the lesion, 2) the orientation, 3) the demarcation of the affected zone and 4) the exact location of the lesion in the concerned anatomical area. Lesion were subsequently classified according to a grading system from 0 to $4(0=$ no similarities, $1=$ some similarities; $\sim 1$ items positive, $2=$ moderate similarities; $\sim 2$ items positive, $3=$ good similarities, $\sim 3$ items positive; $4=$ excellent similarities; all items positive), corresponding to the grade of similarity with the distribution pattern of our segmental vitiligo lesions presenting on the same anatomic location. The assessment was done by 3 investigators (NvG, RS, EM). Lesions were re-evaluated if there was disagreement between the 3 
investigators. In remaining doubtful cases, lesions were classified as "unclassifiable" and were excluded from analysis.

\section{Statistical analysis}

All statistical analyses were performed using SPSS 20.0 for Windows. For comparison between ordinal variables, the linear-by-linear chi square test was used. In case of dichotomous variables, Fisher's exact test was performed. For all tests p values of less than 0.05 were considered to indicate statistical significance.

\section{Results}

\section{Study population}

(1) Segmental vitiligo: In total 254 pictures from lesions located at different body areas of 181 segmental vitiligo patients were enrolled in this study (Table 1). In 152 of them an informed consent was obtained, while 29 patients did not refuse the use of their available data from their medical file (the 'opting out' system). One patient refused the use of the available data. Important clinical data of all included segmental vitiligo patients are shown in table 1 . The majority $(69.2 \%)$ of segmental vitiligo lesions was present in the head and neck area. Halo naevi were present in $26(14.4 \%)$. Congenital naevi were observed in $20(15.7 \%)$ patients.

(2) Control lesions: In total 780 'control' lesions were initially included. In 52.2\% ( $\mathrm{n}=407)$ these lesions were collected by the internet, $25.8 \%(\mathrm{n}=201)$ by medical literature (journals, books) and in $22.1 \%(\mathrm{n}=172)$ it concerned patients seen at our department. In the latter group 147 patients $(85.5 \%)$ did not refuse the use of their pictures from their medical files and could be included for analyses. Twenty-five patients (14.5\%) elected for "opting out" or were excluded due to other reasons (e.g. unknown contact details...). Fifty pictures from books or internet were additionally excluded as they were insufficiently clear for adequate analysis. So, 
in total 724 control lesions could be included for evaluation. Details with respect to type, number and location of all control lesions are summarized in table 2. The highest number of pictures were found for capillary malformations $(n=146)$, herpes zoster $(n=75)$ and naevoid hypomelanosis $(\mathrm{n}=71)$ (Table 2$)$.

\section{Grading of correspondence compared to segmental vitiligo lesions}

Overall, most control lesions $(63.1 \% ; \mathrm{n}=445)$ could be categorized as having a grade $\mathbf{0}$ for similarity. In $32.5 \%(n=229)$ of the control lesions some to moderate grade of correspondence was observed (grade 1-2). Only in a minority of patients $(4.4 \% ; \mathrm{n}=31)$, clear similarities were seen with segmental vitiligo (grade 3-4), while excellent similarity (grade 4) was only found in 5 cases $(0.7 \%$ ).

Statistical analyses using the linear-by-linear association which takes the ordering of categories into account showed higher similarity scores for segmental lentiginosis $(\mathrm{P}<0.001)$, verrucous epidermal naevus $(\mathrm{P}=$ N.S.). Significantly lower similarity scores were observed in ILVEN $(\mathrm{P}=0.025)$, Becker's naevus $(\mathrm{P}=0.001)$, "hypomelanosis of Ito" $(\mathrm{P}=0.049)$ and incontinentia pigmenti $(\mathrm{P}=$ 0.014) compared to the other skin disorders.

Moderate to excellent agreement (grade 2-4) was found in 104 patients (14.8\%). Lesions on the face, neck and the trunk showed most overlap whereas this was less frequently observed on the extremities $(\mathrm{P}=0.011)$. Grade 2 to 4 correspondence was detected more frequently in segmental lentiginosis $(14 / 19,73.7 \%)(\mathrm{P}<0.001)$, naevus sebaceus $(2 / 7 ; 28.6 \%)(\mathrm{P}=\mathrm{N} . \mathrm{S}$.$) , neurofibromatosis type 1(5 / 22$, 22.7\%) (P = N.S.), herpes zoster $(16 / 75,21.3 \%)(P=$ N.S. $)$ and verrucous epidermal naevus $(11 / 56$; $19.6 \%$ ) $(\mathrm{P}=$ N.S.) (Figure 1). Compared to the other skin disorders less grade 2-4 agreement was observed for Becker's naevus (1/40; 2.5\%), segmental Darier's disease (0/15), incontinentia pigmenti (0/14), ILVEN (0/15), "hypomelanosis of Ito" (0/9), linear and whorled hypermelanosis (0/7), segmental Hailey-Hailey disease (0/5) and linear lichen sclerosus (0/4).

Clear correspondence (grade 3-4) was increasingly found in patients with segmental lentiginosis (7/19, $36.8 \%)(\mathrm{P}<0.001)$, verrucous epidermal naevus $(7 / 56,12.5 \%)(\mathrm{P}=0.008)$, segmental lichen planus 
$(2 / 19,10.5 \%)(\mathrm{P}=$ N.S. $)$, and segmental neurofibromatosis type $1(2 / 22 ; 9.1 \%)(\mathrm{P}=$ N.S. $)$ (Figure 2$)$. No obvious similarities (grade 3-4) were found in ILVEN, naevus sebaceus, phacomatosis pigmentokeratotica, macular hyperpigmentation, linear and whorled naevoid hypermelanosis, Becker's naevus, "hypomelanosis of Ito", incontinentia pigmenti, CHILD, cutaneous leiomyomatosis, segmental Darier's disease, segmental Hailey-Hailey disease, linear porokeratosis, lichen striatus, linear lichen sclerosus and linear morphea.

When evaluating the similarity scores according to 6 different groups of cell origin, the melanocyte subgroup was significantly associated with a higher frequency of grade 3-4 similarities $(P=0.031)$. Complete agreement $(n=5)$ was only found in this category $(\mathrm{P}=0.004): 2$ cases of segmental lentiginosis, 1 case of macular hypermelanosis, 1 case of segmental neurofibromatosis type I (café-aulait macule), and 1 case of naevoid hypomelanosis.

A typical recurring pattern for segmental vitiligo could mainly be observed on the face and on the upper part of the trunk, while more variation in distribution was observed at extremities and lower part of the trunk. This typical pattern was mainly seen at the medial side of eyebrow (turned V-shape; Figure 3) and at the midline of the ventral upper thorax (V-shape; Figure 4 ). These patterns were observed in respectively 12 of $26(46.2 \%)$ lesions localized on the forehead and 13 of $28(46.4 \%)$ lesions on the upper half of the trunk. Segmental vitiligo patients with or without congenital naevi or halo naevi had a comparable percentage of overlap with other skin diseases.

\section{Discussion}

The current study assesses the distribution pattern of segmental vitiligo, as the origin of this typical pattern is still questioned. So far, 2 distribution patterns have been suggested in literature to explain its origin: dermatomal and blaschkolinear. However, recent observational studies, demonstrated that the majority of segmental lesions do not show a strictly dermatomal or blaschkolinear pattern. ${ }^{2,11} \mathrm{We}$ therefore decided to evaluate the distribution pattern of segmental vitiligo lesions more in detail. To

our knowledge, this is the first study that investigated the possible overlap between a high number of 
unilateral or band shaped dermatoses and segmental vitiligo in a systematic way. The comparison of these different patterns can provide clues to the pathogenesis of segmental vitiligo. However, we should keep in mind that we can't rule out the possible existence of different or combined etiopathological pathways (e.g. neural, skin homing and mosaicism) underlying the same clinical presentation of segmental vitiligo. ${ }^{1}$

Our study results are in line with our previous observations. ${ }^{2}$ The majority of segmental vitiligo lesions were not strictly dermatomal nor strictly blaschkolinear. The segmental vitiligo pattern seems to be rather unique, although $36.9 \%$ of the control lesions showed similarities (grade 1-4) with segmental vitiligo. The distribution pattern of segmental vitiligo lesions was not statistically different between patients with or without associated halo naevi or congenital naevi. However it remains difficult to draw definite conclusions due to the insufficient patient population size of these two specific subgroups. A further limitation of the present study was that a certain degree subjectivity can be found by the evaluation of similarity scores. To improve this, evaluation of the pictures was performed by 3 different investigators.

Some observation in the past supported the neural hypothesis in segmental vitiligo. ${ }^{4} \mathrm{Wu}$ et al. provided evidence that a dysfunction of the sympathetic nerves play a role in the pathogenesis of the segmental type of vitiligo. They demonstrated an increased cutaneous blood flow compared to the contralateral normal skin and an elevated $\alpha$ - and $\beta$-adrenoceptor response in lesional segmental vitiligo skin. ${ }^{3}$ Moreover, the unilateral distribution of segmental vitiligo with demarcation at the midline has some similarities with diseases where neural cells play a role such as herpes zoster. We found indeed in our study an increased grade 2-4 similarity for herpes zoster. However, when grade 3-4 agreement was taken into account only 2/75 (2.7\%) cases reached grade 3 similarity, whereas no complete agreement (grade 4) between herpes zoster and segmental vitiligo was found (Figure 2). This suggests that the segmental vitiligo pattern has clear differences with herpes zoster although some rough overlap exists. This may explain the confusion in the past with respect to a possible dermatomal distribution.

The most striking similarities were found in mosaic skin disorders, especially in segmental lentiginosis and verrucous epidermal naevus. For these indications a significant increased presence of grade 3-4 
similarity was observed (Figure 2). Segmental lentiginosis is characterized by small, discrete lentigines that are grouped within an area of normal skin, without a hyper- or hypopigmented background. The absence of a background pigmentation is the main difference compared to naevus spilus maculosus. It has been reported in several publications under various terms including partial unilateral lentiginosis (PUL), lentiginous mosaicism, unilateral lentigines, agminated lentiginosis and zosteriform lentiginous naevus. ${ }^{12,13}$ It has been found to be arranged in a flag-like or checkerboard pattern. For similar lesions that follow the Blaschko lines, Happle et al. proposed to use the term naevus lentiginosus linearis (linear lentiginous naevus). ${ }^{14}$ They described this in 2010 as a new entity. An verrucous epidermal naevus is a hamartoma originating from the pluripotent germinative cells located in the basal layer of the embryonic epidermis which have a predominant component of keratinocytes. ${ }^{15}$ Similar to segmental vitiligo, genetic mosaicism has been proposed as a possible underlying mechanism in verrucous epidermal naevus. ${ }^{6}$ Melanocytic-epidermal twin spotting has been described, indicating a common etiological mechanism that must have occurred during early embryogenesis (Boente et al., 2000). ${ }^{16}$

More recently, increasing evidence has been published on the auto-inflammatory theory of segmental vitiligo. ${ }^{17}$ However, it remains elusive whether the deregulated immune system is the primary event (e.g. according to the skin homing theory) or arises secondary to cellular abnormalities in the epidermis (e.g. according to the cutaneous mosaicism theory). ${ }^{1,18}$ A possible analogous example of such a phenomenon has been termed "Blaschkitis" and in children lichen striatus which is an inflammatory dermatitis that has been related to genetic mosaicism. ${ }^{19,20}$ They both present in a Blaschko-linear reaction pattern. It has been suggested that lichen striatus may result from an abnormal cell clone with susceptibility to inflammation that leads to its deletion early in life, whereas a persistent clone with subtle alterations in antigenicity or propensity for inflammatory stimulation might underlie blaschkitis. 
In vitiligo, melanocytes are assumed to be the primary affected cell type. In our study, skin disorders originating from melanocytes were significantly linked to increased grade 3-4 similarities with the segmental vitiligo pattern (Figure 5 and 6). Complete agreement (grade $4 ; n=5$ ) was also only found in mosaic skin disorders involving melanocytes.

We observed in a significant part of our segmental vitiligo lesions a recurring pattern, that seems to be typical for segmental vitiligo (Figure 3 and 4). On the upper trunk and forehead this was often in a "Vshape". Remarkably, this turned V-shape on the forehead seems in accordance to the embryogenic distribution of neural crest cells. This remarkable cutaneous pattern has also been reported by Hann S$\mathrm{K}$ in a case with a naevus spilus on the forehead. ${ }^{21}$ Although our results are based on a significant number of patients, the generalisability of our observations has to be evaluate in future studies. Up to now Happle et al. described 5 different patterns reflecting mosaicism. ${ }^{8,9}$ These patterns are: Blaschko lines (type 1a narrow and type $1 \mathrm{~b}$ broad lines), blocklike (type 2), phylloid (type 3), large patches without midline separation (type 4), and lateralization (type 5). According to our observations segmental vitiligo has no clear similarities with type 1a (narrow Blaschko lines), as reflected in our negative similarity scores for dermatoses with a typical narrow Blaschko linear distribution ("hypomelanosis of Ito", incontinentia pigmenti, linear and whorled hypermelanosis), nor with the type 3-5 patterns of mosaicism. However, similarities with the type $1 \mathrm{~b}$ pattern were observed in the face for some lesions located at the periauricular, mandibular and temporal region (Figure 6). Furthermore, the distribution of V-shaped lesion on the upper part of the trunk resembled a combination of the type $1 \mathrm{~b}$ and type 2 pattern (Figure 4 ). However, as the majority of our segmental vitiligo lesions has no clear similarities with the currently defined 5 patterns of mosaicism we might speculate that the "segmental vitiligo pattern" probably represent a distinct and new ("type 6") pattern of cutaneous mosaicism.

According to the theory of Happle, one can hypothesize that a mixed type of vitiligo (combination of segmental and non segmental vitiligo) could represent a superimposed form of segmental mosaicism, due to postzygotic loss of heterozygosity involving one of the genes predisposing the disorder, while the solitary segmental lesions can be classified as an 'isolated' mosaic form. The concept of 
superimposed segmental vitiligo is however still a theory and convincing data at the molecular level are missing. ${ }^{7}$

In conclusion our study provides additional evidence that segmental vitiligo has probably been classified incorrectly in the past to a dermatomal distribution. Based on our clinical observation of segmental vitiligo lesions, a rather distinct pattern was found, not entirely corresponding to other unilateral or band shaped dermatoses. However, some skin disorders of melanocytic origin (such as segmental lentiginosis) clearly have more overlap with segmental vitiligo compared to other linear dermatoses. This observation supports the possible role of cutaneous mosaicism for at least a part of the segmental vitiligo lesions.

\section{Acknowledgements}

We wish to express our sincere gratitude to Dr. Sandra Toelle, Department of Neurology, University Children's Hospital, Zurich, Switzerland, for allowing us to use pictures from her publication. She also arranged the consent of the patient involved. We also would like to thank very much all the patients

that gave permission to use their data and images in this study. Further thanks goes to Prof. Dr. R. Happle (Philipp University of Marburg, Germany) for providing many helpful suggestions and interesting editorial comments. We also thank Peter Batsleer, Department of Dermatology, Ghent University Hospital, Ghent Belgium for providing technical assistance with digital images. This research was supported by a research grant to R. Speeckaert from the Research Foundation (No. BOF10/doc/403), Ghent University and to N. van Geel from the Scientific Research FoundationFlanders (FWO Senior Clinical Investigator). 
Table 1: Clinical characteristics of the segmental vitiligo study population

\begin{tabular}{|l|l|}
\hline Characteristic & \\
\hline Number of lesions (different body locations) & 254 \\
\hline Number of patients & 181 \\
\hline Age of onset (mean; yrs) & 18 (IQR: 8 -27) \\
\hline Age at inclusion (mean; yrs) & 29 (IQR: 17-38) \\
\hline Men (\%) & $78 / 181(43.1 \%)$ \\
\hline Affected body area: & \\
\hline Arm & $30 / 181(16.6 \%)$ \\
\hline Leg & $16 / 181(8.8 \%)$ \\
\hline Face & $110 / 181(60.8 \%)$ \\
\hline Trunk & $44 / 181(24.3 \%)$ \\
\hline Autoimmune diseases & $54 / 181(29.8 \%)$ \\
\hline Family history of vitiligo & $17 / 148(11.5 \%)$ \\
\hline Halo naevus & $27 / 152(17.8 \%)$ \\
\hline Congenital naevus & $26 / 179(14.5 \%)$ \\
\hline
\end{tabular}

Table 2: Distribution of the control lesions

\begin{tabular}{|l|l|l|l|l|}
\hline Disease & Total frequency & Face and neck & Extremities & Trunk \\
& $\mathbf{N}(\%)$ & $\mathbf{N}(\%)$ & $\mathbf{N}(\%)$ & $\mathbf{N}(\%)$ \\
\hline Herpes zoster & $75(10.4)$ & $31(41.3)$ & $6(8.0)$ & $38(50.7)$ \\
\hline Capillary malformation & $146(20.2)$ & $102(69.8)$ & $17(11.6)$ & $27(18.5)$ \\
\hline Verrucous epidermal naevus & $56(7.7)$ & $10(17.9)$ & $16(28.5)$ & $30(53.6)$ \\
\hline ILVEN & $15(2.1)$ & $1(6.7)$ & $13(86.7)$ & $1(6.7)$ \\
\hline
\end{tabular}




\begin{tabular}{|c|c|c|c|c|}
\hline Naevus sebaceus & $7(1.0)$ & $6(85.7)$ & / & $1(14.3)$ \\
\hline $\begin{array}{l}\text { Phacomatosis } \\
\text { pigmentokeratotica }\end{array}$ & $11(1.5)$ & $6 / 11(54.6)$ & $1(9.1)$ & $4(36.4)$ \\
\hline Macular hyperpigmentation & $34(4.7)$ & $4(11.7)$ & $6(17.7)$ & $24(70.6)$ \\
\hline $\begin{array}{l}\text { Linear and whorled naevoid } \\
\text { hypermelanosis }\end{array}$ & $9(1.2)$ & / & $1(11.1)$ & $8 / 9(88.9)$ \\
\hline Segmental lentiginosis & $25(3.5)$ & $9(36.0)$ & $12(48.0)$ & $4(16.0)$ \\
\hline Naevus spilus & $42(5.8)$ & $9(20.5)$ & $10(23.8)$ & $23(54.8)$ \\
\hline Becker's naevus & $40(5.5)$ & $1(2.5)$ & $7(17.5)$ & $32(80.0)$ \\
\hline $\begin{array}{l}\text { Segmental neurofibromatosis } \\
\text { type I }\end{array}$ & $22(3.0)$ & $6(27.3)$ & $1(4.5)$ & $15(68.2)$ \\
\hline Naevoid hypomelanosis & $71(9.8)$ & $24(33.8)$ & $18(25.3)$ & $29(40.8)$ \\
\hline "Hypomelanosis of Ito" & $9(1.2)$ & / & $8(88.9)$ & 1 (11.1) \\
\hline Incontinentia pigmenti & $14(1.9)$ & / & $5(35.7)$ & $9(64.3)$ \\
\hline CHILD & $3(0.4)$ & / & / & $3(100.0)$ \\
\hline Cutaneous leiomyomatosis & $16(2.2)$ & $1(6.3)$ & $2(12.5)$ & $13(81.3)$ \\
\hline Segmental Darier's disease & $15(2.1)$ & $1(6.7)$ & $5(33.3)$ & $9(60.0)$ \\
\hline Segmental Hailey-Hailey & $5(0.7)$ & / & $2(40.0)$ & $3(60.0)$ \\
\hline Linear Lichen planus & $19(2.6)$ & $5(26.3)$ & $7(36.8)$ & $7(36.8)$ \\
\hline Linear Porokeratosis & $11(1.5)$ & $1(9.1)$ & $7(63.6)$ & $3(27.3)$ \\
\hline Lichen striatus & $37(5.1)$ & $2(5.4)$ & $24(64.9)$ & $11(29.7)$ \\
\hline Linear lichen sclerosus & $4(0.6)$ & $1(25.0)$ & $1(25.0)$ & $2(50.0)$ \\
\hline Linear morphea & $38(5.2)$ & $27(71.1)$ & $8(21.1)$ & $3(7.9)$ \\
\hline
\end{tabular}




\section{References}

1 van Geel N, Mollet I, Brochez L et al. New insights in segmental vitiligo: case report and review of theories. Br J Dermatol 2012; 166: 240-6.

2 van Geel N, De Lille $S$, Vandenhaute $S$ et al. Different phenotypes of segmental vitiligo based on a clinical observational study. J Eur Acad Dermatol Venereol 2011; 25: 673-8.

3 Wu CS, Yu HS, Chang HR et al. Cutaneous blood flow and adrenoceptor response increase in segmental-type vitiligo lesions. J Dermatol Sci 2000; 23: 53-62.

$4 \quad$ Nelhaus G. Acquired unilateral vitiligo and poliosis of the head and subacute encephalitis with partial recovery. Neurology 1970; 20: 965-74.

5 Koga M, Tango T. Clinical features and course of type A and type B vitiligo. Br J Dermatol 1988; 118: 223-8.

6 Taieb A, Morice-Picard F, Jouary T et al. Segmental vitiligo as the possible expression of cutaneous somatic mosaicism: implications for common non-segmental vitiligo. Pigment Cell Melanoma Res 2008; 21: 646-52.

7 Happle R. Superimposed segmental manifestation of polygenic skin disorders. J Am Acad Dermatol 2007; 57: 690-9.

8 Happle R. [Patterns on the skin. New aspects of their embryologic and genetic causes]. Hautarzt 2004; 55: 960-1, 4-8.

9 Happle R. Mosaicism in human skin. Understanding the patterns and mechanisms. Arch Dermatol 1993; 129: 1460-70.

10 Happle R, Assim A. The lines of Blaschko on the head and neck. J Am Acad Dermatol 2001; 44: 612-5.

11 Kim DY, Oh SH, Hann SK. Classification of segmental vitiligo on the face: clues for prognosis. BrJ Dermatol 2011; 164: 1004-9.

12 Toelle SP, Boltshauser E, Wirth MG et al. Association of lentiginous mosaicism and congenital cataract in a girl. Eur J Dermatol 2006; 16: 360-2.

13 Micali G, Nasca MR, Innocenzi D et al. Agminated lentiginosis: case report and review of the literature. Pediatr Dermatol 1994; 11: 241-5.

14 Happle R, Metze D, Vera Casano A. Naevus lentiginosus linearis: A distinct skin disorder. Acta Derm Venereol 2010; 90: 210-1.

15 Rogers M. Epidermal nevi and the epidermal nevus syndromes: a review of 233 cases. Pediatr Dermatol 1992; 9: 342-4.

16 Boente MC, Pizzi de Parra N, Larralde de Luna M et al. Phacomatosis pigmentokeratotica: another epidermal nevus syndrome and a distinctive type of twin spotting. Eur J Dermatol 2000; 10: 190-4.

17 van Geel NA, Mollet IG, De Schepper S et al. First histopathological and immunophenotypic analysis of early dynamic events in a patient with segmental vitiligo associated with halo nevi. Pigment Cell Melanoma Res 2010; 23: 375-84.

18 Gauthier Y, Cario Andre M, Taieb A. A critical appraisal of vitiligo etiologic theories. Is melanocyte loss a melanocytorrhagy? Pigment Cell Res 2003; 16: 322-32.

19 Taieb A, el Youbi A, Grosshans E et al. Lichen striatus: a Blaschko linear acquired inflammatory skin eruption. J Am Acad Dermatol 1991; 25: 637-42.

20 Lipsker D, Cribier B, Girard-Lemaire F et al. Genetic mosaicism in an acquired inflammatory dermatosis following the lines of Blaschko. Arch Dermatol 2000; 136: 805-7.

21 Hann SK. Particular clinical characteristics of segmental vitiligo. In: Vitiligo (Taieb A, Picardo M, eds), $1^{\text {st }}$ edn. Springer-Verlag 2010; 296-298. 
Legends

Figure 1: Good to complete (grade 2-4) similarities in the different skin diseases

Figure 2: Almost complete to complete (grade 3-4) similarities in the different skin diseases

Figure 3: (a) Typical pattern of segmental vitiligo on the forehead at the medial side of the eyebrow (turned V-shape) in our patient population (b) Overlap of all unilateral lesions above on the right (c) Overlap of all unilateral lesions above on the left (d) Combination of all lesions above.

Figure 4: Typical pattern of segmental vitiligo on the upper part of the trunk (V-shape) in our patient population

Figure 5: Clinical pictures of a patient with lentiginous lesions on the front (a) and back (c) of the trunk (pictures on the left) corresponding to the depigmentation pattern in a patient with segmental vitiligo in the same anatomic regions (pictures on the right). One picture (d) with additional UV light.

Figure 6: Clinical pictures of lentiginous lesions on the face and the upper arm (pictures on the left) corresponding to segmental vitiligo (pictures on the right).

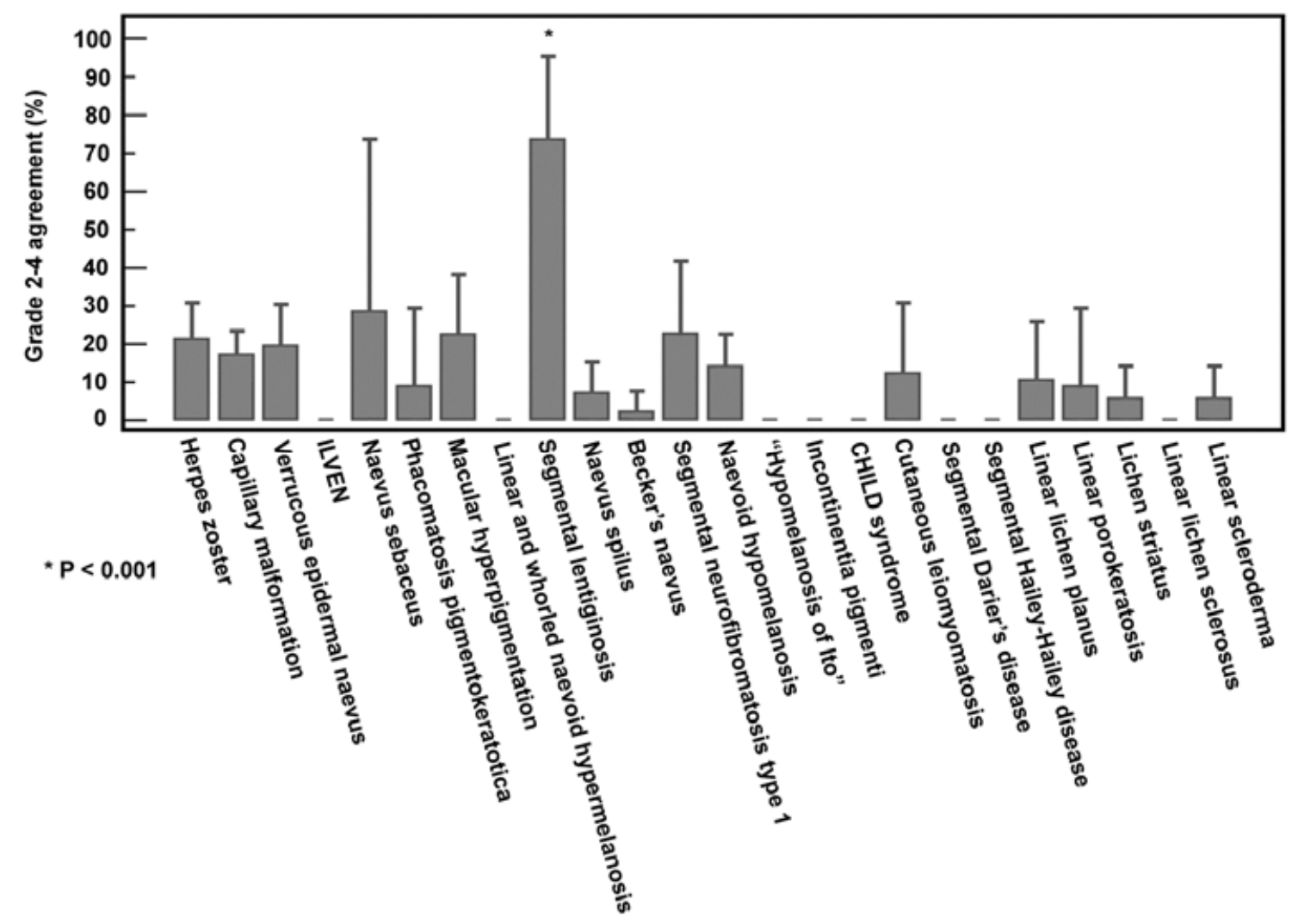




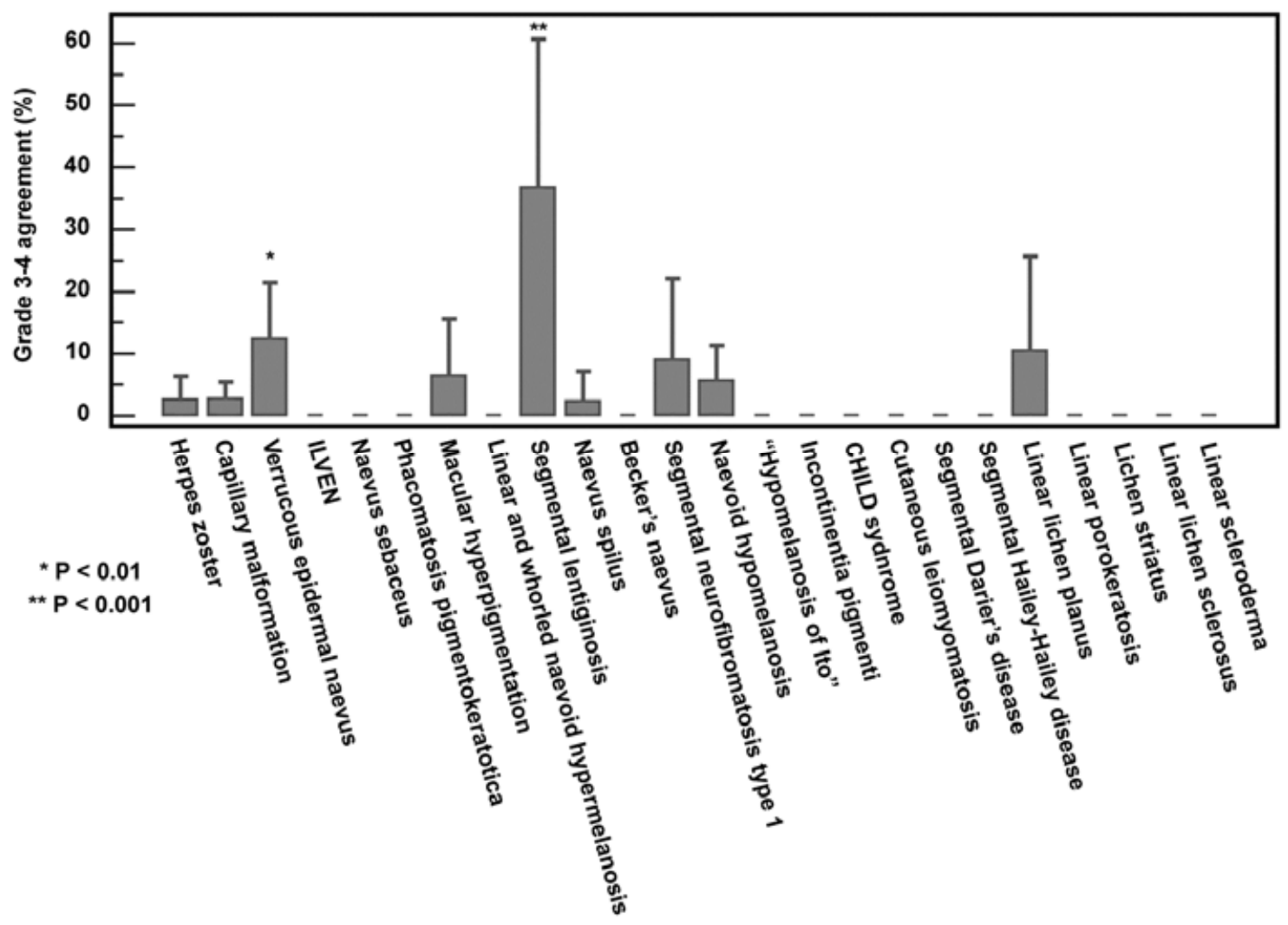


(a)
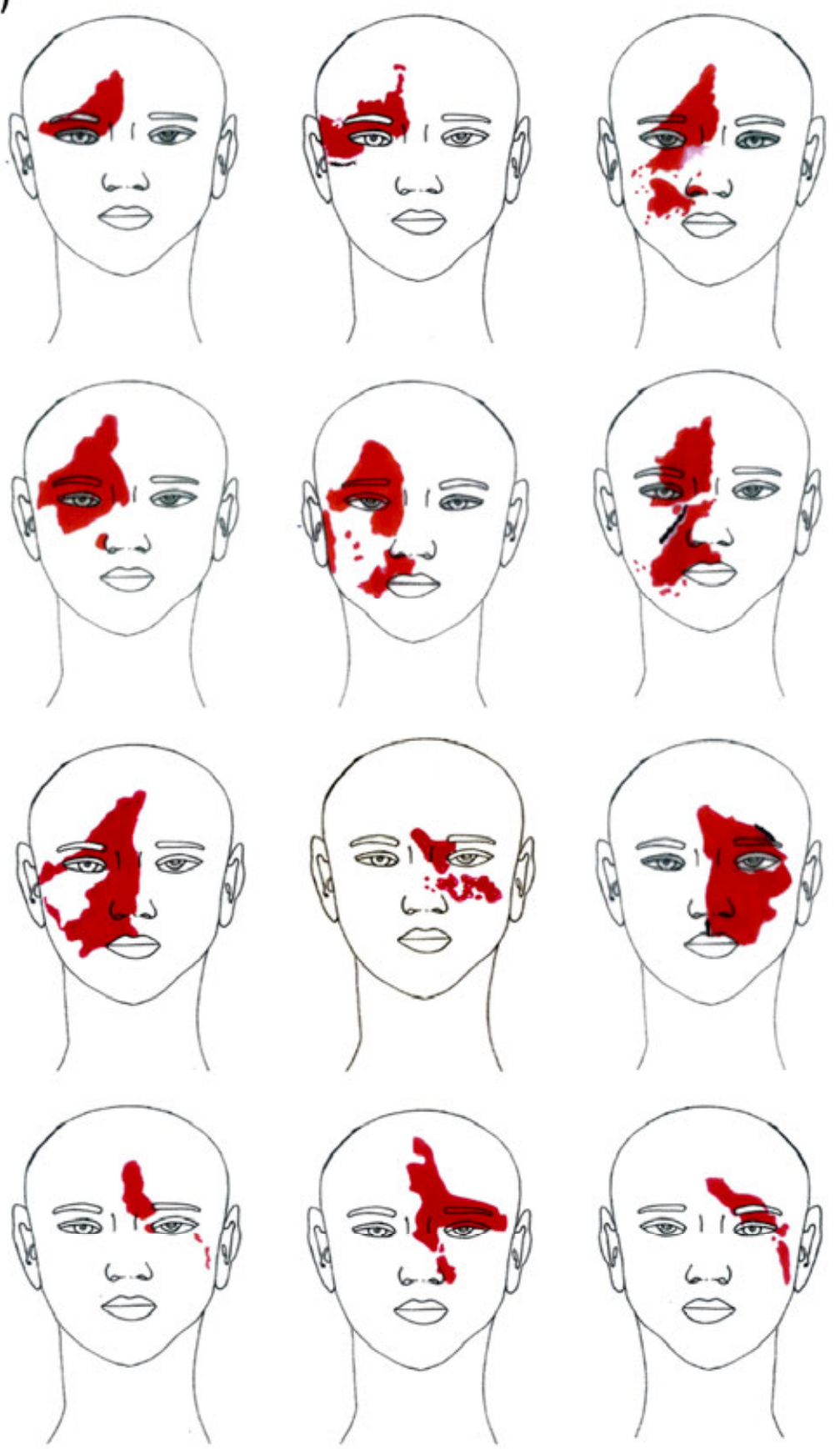

(b)

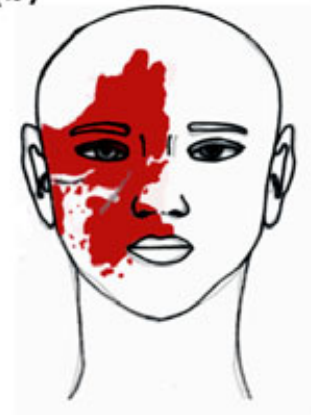

(c)

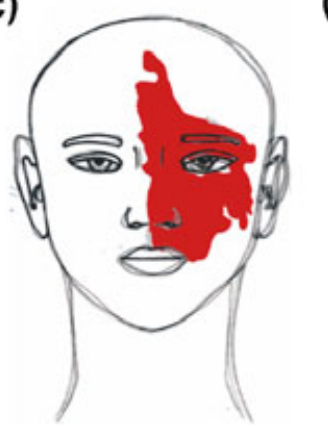

(d)

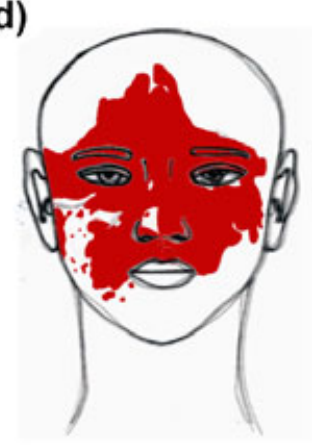



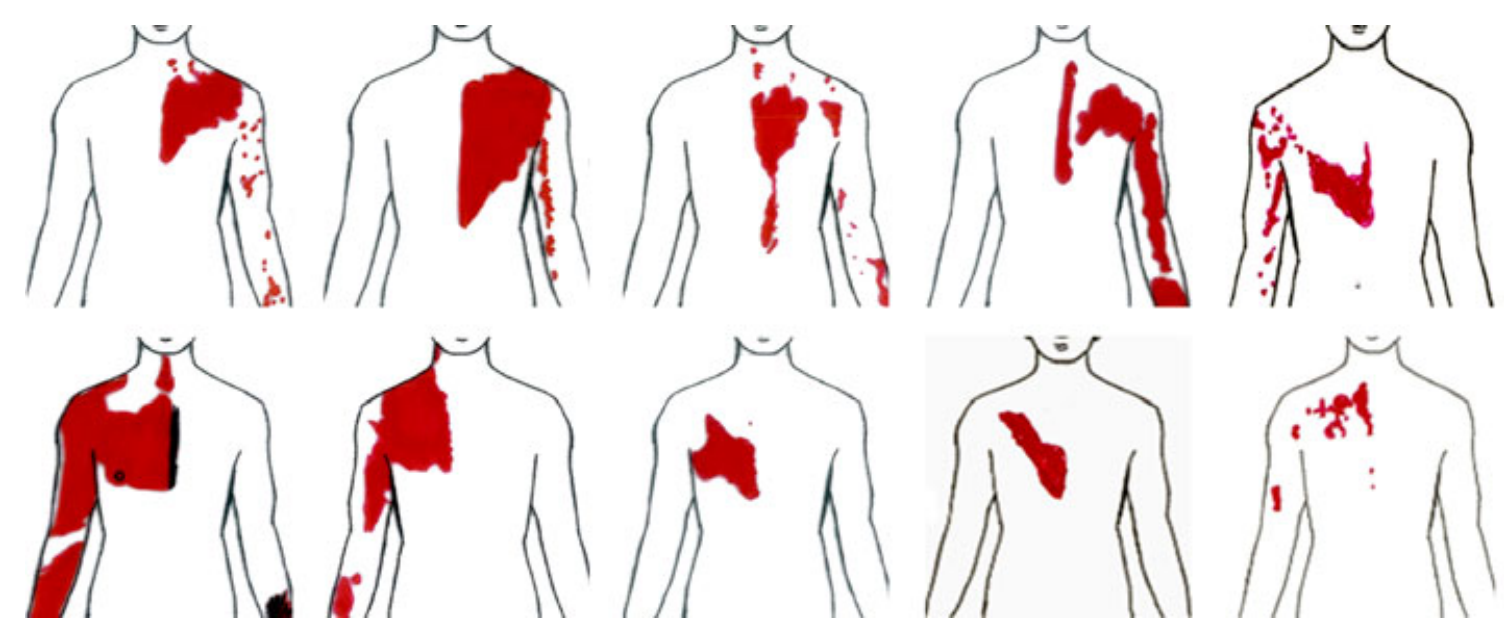

(a)

(b)
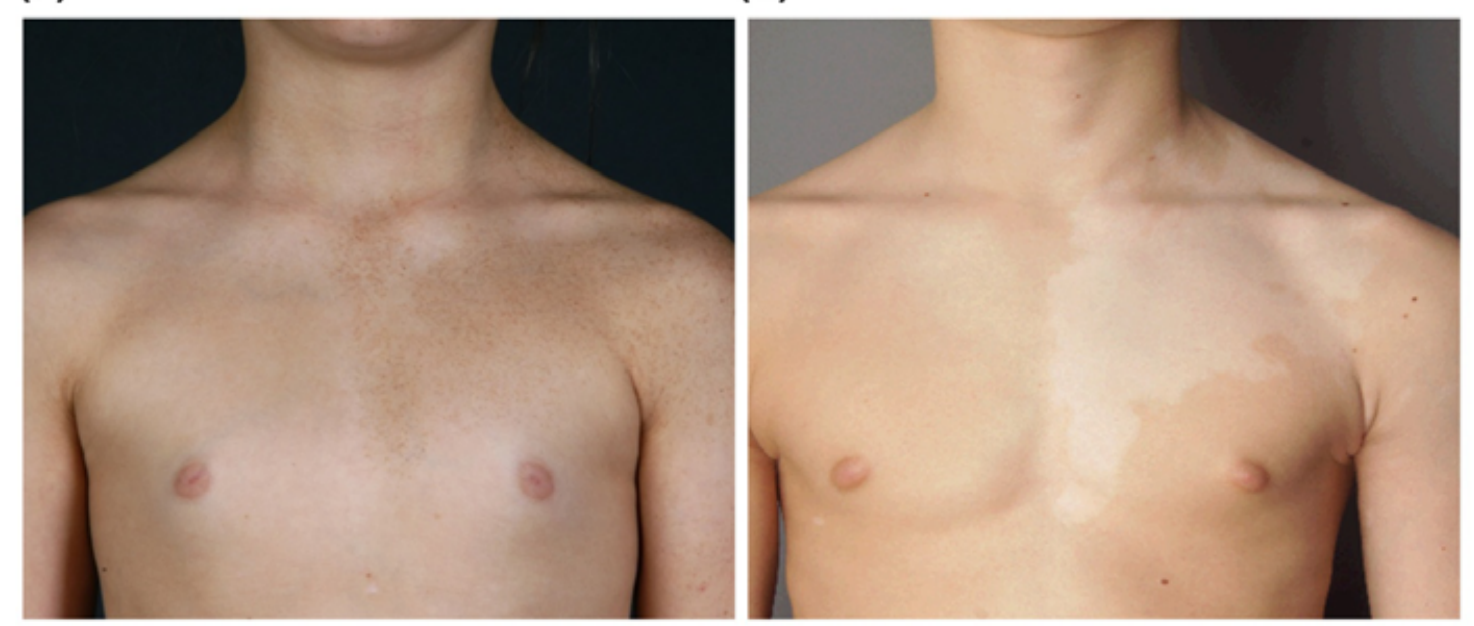

(c)

(d)

(e)

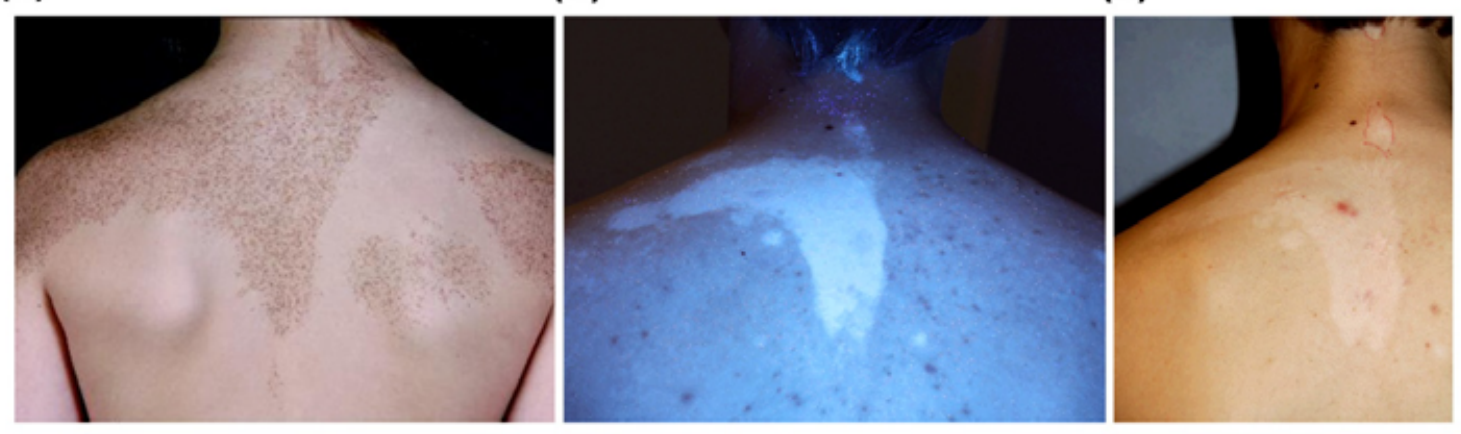


(a)

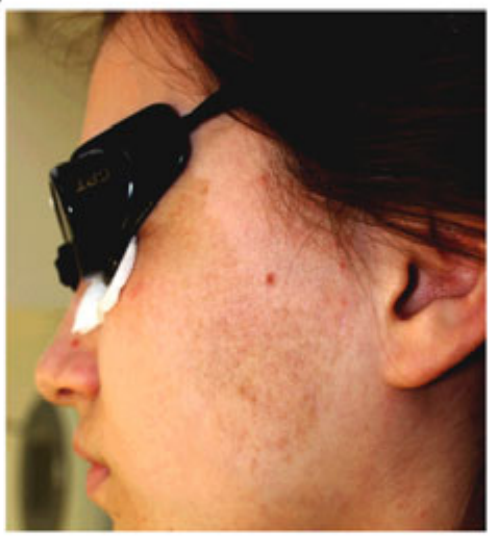

(c)

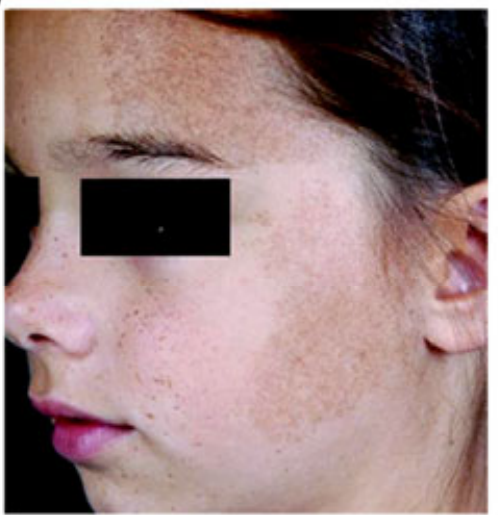

(e)

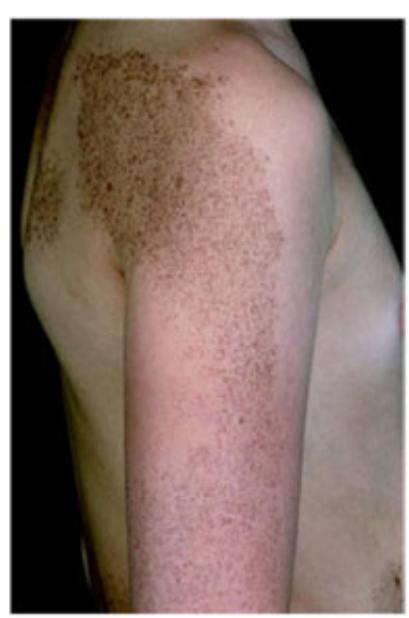

(b)

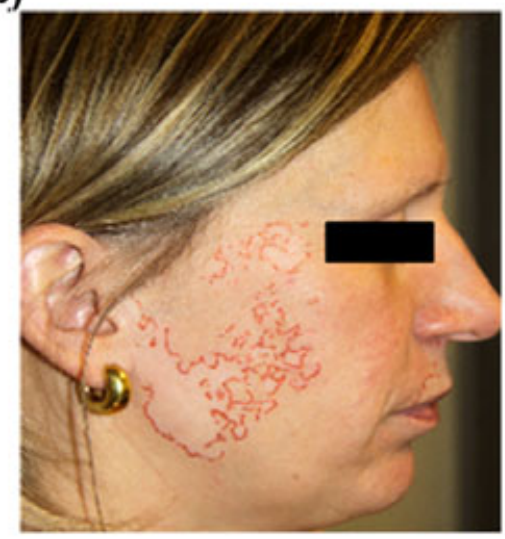

(d)

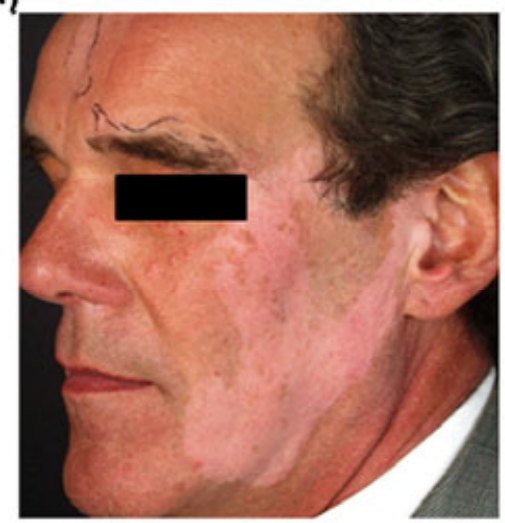

(f)

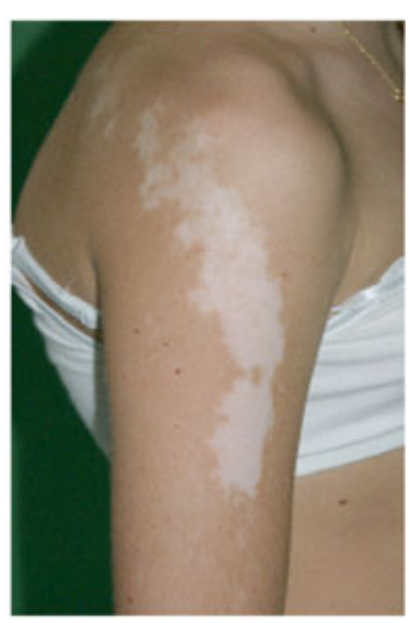

